\title{
Sistema hipermedia basado en competencias para el diagnóstico del aprendizaje de fracciones matemáticas (SMCDAFRAC)
}

\author{
E. Erica Vera Cervantes ${ }^{1,2}$, Carmen Cerón Garnica ${ }^{1}$, Yadira Navarro ${ }^{1}$ y \\ María Magdalena Ortiz Funez ${ }^{1}$ \\ ${ }^{1}$ Benemérita Universidad Autónoma de Puebla \\ Facultad de Ciencias de la Computación \\ Avenida San Claudio, 14 Sur, Ciudad Universitaria \\ ${ }^{2}$ Centro de Estudios Superiores en Educación (CESE) \\ Puebla, México \\ \{eevclibra, academicaceron, ynavarro44\}@gmail.com
}

\begin{abstract}
Resumen. A partir de la Reforma Integral de Educación Básica, se estableció el Plan de estudios 2011, en el cual se definen las competencias para la vida, el perfil de egreso, los estándares curriculares y los aprendizajes esperados en los alumnos de este nivel. El propósito de este artículo es presentar el diseño y desarrollo del Sistema Hipermedia Adaptativo (SHA) el cual considera las características del estudiante (perfil), para adaptar y presentar los contenidos basados en competencias para apoyar a desarrollar las habilidades en las matemáticas, en el tema de fracciones de acuerdo a los programas de estudio vigentes. Finalmente se presentan los resultados obtenidos del sistema al realizar una prueba piloto con una muestra de alumnos de tercer grado de primaria.
\end{abstract}

Palabras clave: Competencias, sistema hipermedia adaptativo, multimedia y fracciones.

\section{Introducción}

Las instituciones educativas han incorporado el uso de las Tecnologías de Información y Comunicación (TIC) con una tendencia en el enfoque constructivista donde lo importante es aprender a aprender, para usar el objeto de conocimiento y saber dónde encontrar la información adecuada para la solución de problemas determinados [1]. Las TIC han proporcionado mayor velocidad, alcance y acceso a distintos recursos de información. Actualmente se requiere sistemas de información tecnológicos que permitan una personalización y adaptación de la información a las necesidades específicas de aprendizaje de cada alumno de tal forma que en el campo de la educación el sistema permita ayudar a planificar el proceso educativo de acuerdo a los contenidos para tomar decisiones para mejorar el rendimiento académico de los alumnos. La mayoría de los diseños de los sistemas interactivos permite a los alumnos mayor interactividad, concentrarse en el razonamiento y en la solución de problemas [2]. Es 
decir, que los entornos de aprendizaje posibiliten actividades reales y contextualizadas para los estudiantes.

Actualmente los profesores han identificado una problemática en la enseñanza de las matemáticas al ocupar los métodos tradicionalistas que fomentan un aprendizaje pasivo, memorístico y no reflexivo. A partir de lo anterior existe el interés de incorporar las Tecnologías de Información y Comunicación (TIC) que contribuyan a la generación de ambientes de aprendizaje para propiciar mediante el uso de diversos recursos tecno-pedagógicos el desarrollo de habilidades en alumno.

Este proyecto tiene como propósito el diseño y desarrollo de un Sistema Hipermedia Adaptativo (SHA) denominado SMCDAFRAC (Sistema Multimedia basado en Competencias para el diagnóstico del aprendizaje de Fracciones), el cual surge de la necesidad de involucrar el uso de las TIC y apoyar el proceso de enseñanzaaprendizaje, donde los nuevos planes de estudio de acuerdo a la Reforma Integral de Educación Básica [3], los cuales promueven un aprendizaje por competencias y estrategias de aprendizaje de acuerdo a las necesidades de los alumnos para activar el pensamiento e integrar esos saberes a su desempeño cotidiano. El sistema es un medio para apoyar a los profesores y los alumnos en el campo de las matemáticas, el cual contempla la hipermedia, la adaptabilidad y el contenido educativo mostrando los distintos niveles de saberes/aprendizajes que el alumno debe adquirir de acuerdo a los planes y programas de estudio.

El objetivo del sistema es apoyar a propiciar el pensamiento matemático para el desarrollo de la imaginación, creatividad y el razonamiento lógico. El SHA permite identificar las necesidades de aprendizaje y proponer actividades de aprendizaje que conlleven al desarrollo de competencias en el alumno para la solución de problemas en el tema de fracciones. Esto le ayudará aprender a aprender y reflexionar sobre sus competencias adquiridas para mejorar su desarrollo intelectual y autonomía.

Por lo cual este documento se organiza de la siguiente manera: la fundamentación teórica de los sistemas hipermedias adaptativos, la estructura de los sistemas web y las competencias en las matemáticas se revisan en la sección 2; el análisis y diseño del sistema en UML, la arquitectura cliente-servidor, el diseño de la base de datos, el desarrollo del sistema con tecnología Active Server Pages (ASP) y HTML se presentan en la sección 3; el desarrollo y las pruebas del sistema se muestran en la sección 4 y finalmente en la sección 5 se presentan las conclusiones y el trabajo a futuro de esta investigación.

\section{Marco teórico}

\subsection{Sistema hipermedia adaptativo (SHA)}

La educación ha sido una de las áreas de aplicación más populares en el área de los Sistemas Hipermedia Adaptativos. Las investigaciones que se han realizado en este campo, se han dirigido sobre todo a técnicas y métodos de adaptación para apoyar las necesidades de aprendizaje del usuario, sus intereses, conocimientos previos y brindar 
un gran volumen de información. Según Brusilovsky un Sistema Hipermedia Adaptativo es un sistema basado en hipertexto e hipermedia que refleja algunas características del usuario en el modelo de usuario y aplica este modelo para adaptar varios aspectos visibles del sistema al usuario [4]. Para Gaudioso define los SHA son aquellos sistemas de hipermedia capaces de ajustar su presentación y navegación a las diferencias de los usuarios, reduciendo así los problemas de desorientación y falta de comprensión, propios de los sistemas hipermedia no adaptativos [5]. Un sistema se considera adaptativo cuando se adapta de forma automática y personalizada a las necesidades del usuario [4]. Por lo que el SHA permite personalizar la información almacenada y la presenta a los usuarios según sus preferencias, conocimientos e intereses. El proceso de personalización permite mostrar la información que es apropiada para tipo de conocimiento y aprendizaje de cada usuario [6]. El modelo de adaptación posee un conjunto de reglas que permiten adaptar los contenidos al perfil del usuario. El uso de reglas para establecer la adaptación está inspirada por varias investigaciones sobre los SHA, entre ellas la propuesta por Raad y Causse llamada "Modelización de la adaptación del Sistema Hipermedia basado en reglas Activas" que propone una separación entre la parte del comportamiento y las entidades del modelo que permiten añadir nuevas técnicas [7]. En esta investigación se utilizan reglas de la forma: "Con la ocurrencia de un evento $E$, si la condición $C$ se cumple, entonces el sistema ejecuta una acción A." En general, las reglas permiten al sistema seleccionar adaptativamente, considerando las características del usuario y el tipo de contenido que debe aprender para cumplir con los objetivos, donde se logran manifestar relaciones interesantes a partir de la información existente.

\subsection{Multimedia}

En el ámbito de la computación el término multimedia designa el uso de varios recursos o medios, como audio, video, animaciones, texto e imágenes en una computadora, sin quedarse, sólo, en un collage de medios, al integrar los datos que puede manejar la computadora. La multimedia ofrece posibilidades de creatividad mediante los sistemas de computación. Según Bartolomé, el objetivo de la multimedia aplicada a la educación es alcanzar a desarrollar destrezas y actitudes necesarias en los estudiantes de modo que ellos se puedan comunicar con distinto lenguajes y medios, que además puedan desarrollar autonomía personal y espíritu crítico para formar una sociedad justa y multicultural [8]. La necesidad de incorporar los materiales multimedia en la educación se hace cada vez más latente ya que nos encontramos inmersos en una sociedad del conocimiento y la información; los alumnos demandan, cambios en los procesos de enseñanza para que el aprendizaje sea significativo y resulte motivador para ellos asistir a clases dinámicas, entretenidas y contextualizadas.

\subsection{Competencias matemáticas y resolución de problemas}

Se entienden así las matemáticas como un conjunto de cuestiones y problemas, de ideas y formas de actuar y de tecnologías simbólicas y organizativas que conllevan no 
sólo utilizar cantidades y formas geométricas, sino también hacerse preguntas y resolver problemas, obtener modelos e identificar relaciones y estructuras, de modo que, al analizar los fenómenos y situaciones que se presentan en la realidad, se puedan obtener informaciones y conclusiones que inicialmente no estaban explícitas [8]. Las competencias en matemáticas enfatizan en que el alumno debe ser competente para argumentar, cuantificar, analizar críticamente la información, representar y comunicar, resolver y enfrentarse a problemas, usar técnicas e instrumentos matemáticos e integrar los conocimientos adquiridos [9]. La Reforma Integral de Educación Básica en su enfoque por competencias [3] afirma que "La resolución de problemas es el mejor camino para desarrollar estas competencias ya que es capaz de activar las capacidades básicas del individuo, como son: leer comprensivamente, reflexionar, establecer un plan de trabajo, revisarlo, adaptarlo, generar una hipótesis, verificar el ámbito de validez de las soluciones, etc. A su vez, posibilita experimentar, particularizar, conjeturar, elegir un lenguaje apropiado, probar una conjetura, generalizar, utilizar distintas partes de las matemáticas, verificar una solución, etc.’[9].

Las competencias enfatizan en la habilidad para seguir determinados procesos de pensamiento (como la inducción y la deducción, entre otros) y aplicar algunos algoritmos de cálculo o elementos de la lógica, lo que conduce a identificar la validez de los razonamientos y a valorar el grado de certeza asociado a los resultados derivados de los razonamientos válidos. Disposición favorable y de progresiva seguridad y confianza hacia la información y las situaciones que contienen elementos o soportes matemáticos, así como hacia su utilización cuando la situación lo aconseja, basadas en el respeto y el gusto por la certeza y en su búsqueda a través del razonamiento ( $1^{\mathrm{a}}$ fase: comprender). Utilizar los elementos y razonamientos matemáticos para enfrentarse a aquellas situaciones cotidianas que los precisan. Por tanto, la identificación de tales situaciones, la aplicación de estrategias de resolución de problemas, y la selección de las técnicas adecuadas para calcular, representar e interpretar la realidad a partir de la información disponible están incluidas en ella (2 $2^{\text {a }}$ fase: pensar) [9].

\section{Análisis y diseño del sistema}

Para el análisis y el diseño del sistema se determinaron los Casos de Uso del sistema en UML [10]. Para Grimon un SHA debe modelar tres partes: el subsistema adaptativo, el subsistema de hipermedia y el modelo de tareas [11].

El modelo del análisis del Sistema Hipermedia Adaptativo contiene los siguientes componentes para su funcionalidad: Modelo del Usuario, Modelo de Adaptación, Modelo de Contenidos y el Modelo de Diagnóstico como se muestra en la Figura 1. 


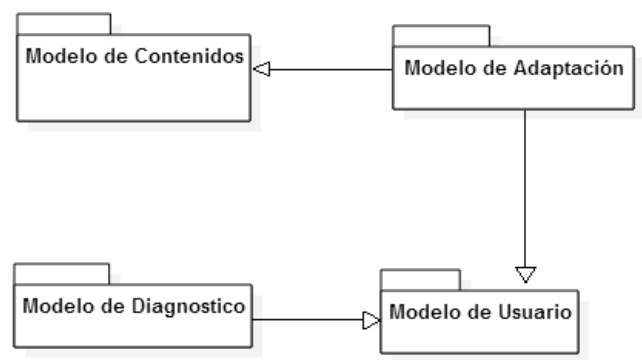

Fig. 1. Modelo de Componentes del Sistema Hipermedia

El sistema permite identificar tres usuarios para el acceso y manipulación del sistema:

- Usuario Administrador: Realizar todos los procesos del sistema como es eliminar, modificar la información y consultas en general.

- Usuario Docente: Puede realizar consultas generales, alta de materiales y modificación con respecto a los contenidos, actividades y evaluación del tema.

- Usuario Alumno: Consulta los contenidos y materiales de información, al realizar el diagnóstico, el sistema adapta y determina los tipos de aprendizajes que requiere dominar y le propone una serie de actividades para el desarrollo de las competencias.

El sistema permite presentar una adaptación para los perfiles de los distintos usuarios, como se muestra en la Figura 2, en el caso del alumno.

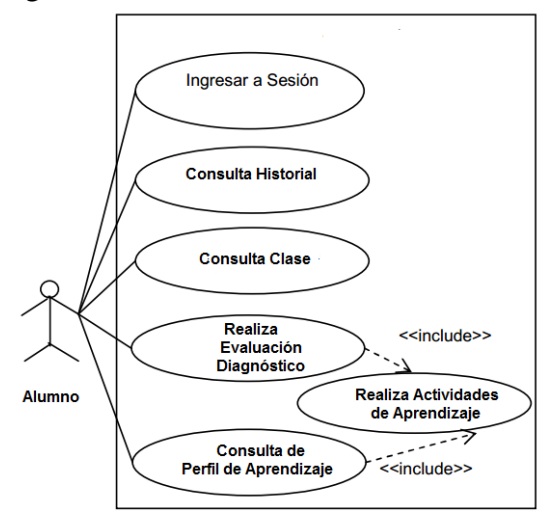

Fig. 2. Diagrama de Caso de Uso del Alumno

\subsection{Diagnóstico académico para la evaluación de los aprendizajes de fracciones}

El sistema presenta una adaptación para los perfiles de los distintos usuarios de acuerdo al Diagnóstico Académico. El procedimiento que se propuso para lograr en el Sistema Hipermedia Adaptativo realiza varias tareas, como son: 
a) Diagnosticar los conceptos o procedimientos que no se aprendieron de Fracciones.

b) Emitir un nivel de evaluación (puntuación) del aprendizaje mostrado por el alumno con respecto al tema de Fracciones.

c) Recomendar medidas para superar problemas y mejorar los aprendizajes.

d) Incorpora fragmentos de autoinstrucción para remediar las deficiencias de aprendizaje diagnosticadas.

\subsubsection{Selección de las unidades y aprendizajes relevantes}

El proceso de evaluación del alumno inicia con la determinación del perfil deseable. En él se incluyen todos los conocimientos y habilidades que se esperan del estudiante, como resultado de haber intervenido en un proceso de enseñanza- aprendizaje y de acuerdo a sus necesidades.

Por lo que la adaptación de las unidades o temas seleccionados se realiza mediante los siguientes tres pasos:

a) Seleccionar las ideas principales o esenciales, o temas relacionados a los números fraccionarios.

b) Determinar los procesos cognitivos deseables para cada idea esencial.

c) Señalar el tipo de aprendizaje.

El aprendizaje se puede clasificar de diferentes formas:

- Indispensable. Es el aprendizaje referido a cuestiones que son básicas para el tema, unidad o área, es decir, conceptos, hechos y procedimientos que el alumno debe tener presentes toda su vida. Las ideas indispensables corresponden a un pequeño grupo dentro de las esenciales.

- Esencial. Es el aprendizaje que abarca todas las ideas principales extraídas del tema, unidad o área.

- Antecedente o componente. Son ideas que corresponden a complementos o antecedentes de la idea esencial para poder responder a los otros aprendizajes.

\subsubsection{Elaboración del diagnóstico para los aprendizajes del tema de los números fraccionarios.}

El método consta de tres pasos:

a) Seleccionar las ideas principales o esenciales.

b) Determinar los procesos cognitivos deseables para cada idea esencial.

c) Señalar el tipo de aprendizaje: indispensable o esencial.

Para cada idea esencial o indispensable se separa en sus componentes o antecedentes. La forma más elemental para definir las ideas esenciales consiste en plantearse la pregunta: ¿Qué debe saber o saber hacer el alumno de este nivel que aprendió el tema en cuestión?, el Sistema está diseñado para evaluar las preguntas de acuerdo a los aprendizajes esperados de las ideas principales [12].

El sistema maneja tres niveles de desempeño de las competencias que son: 1) Inicial, 2) Regular y 3) Excelente. A los usuarios los clasifica de acuerdo a su Perfil en: Experto, Medio y Principiante. Para evaluar los contenidos se maneja en tres puntajes: 1) Bajo, menor a la media, 2) Regular, dentro de la media y 3) Alto, superior a la media. Tal como se muestra en la Figura 3. 
Sistema hipermedia basado en competencias para el diagnóstico del aprendizaje de fracciones ...

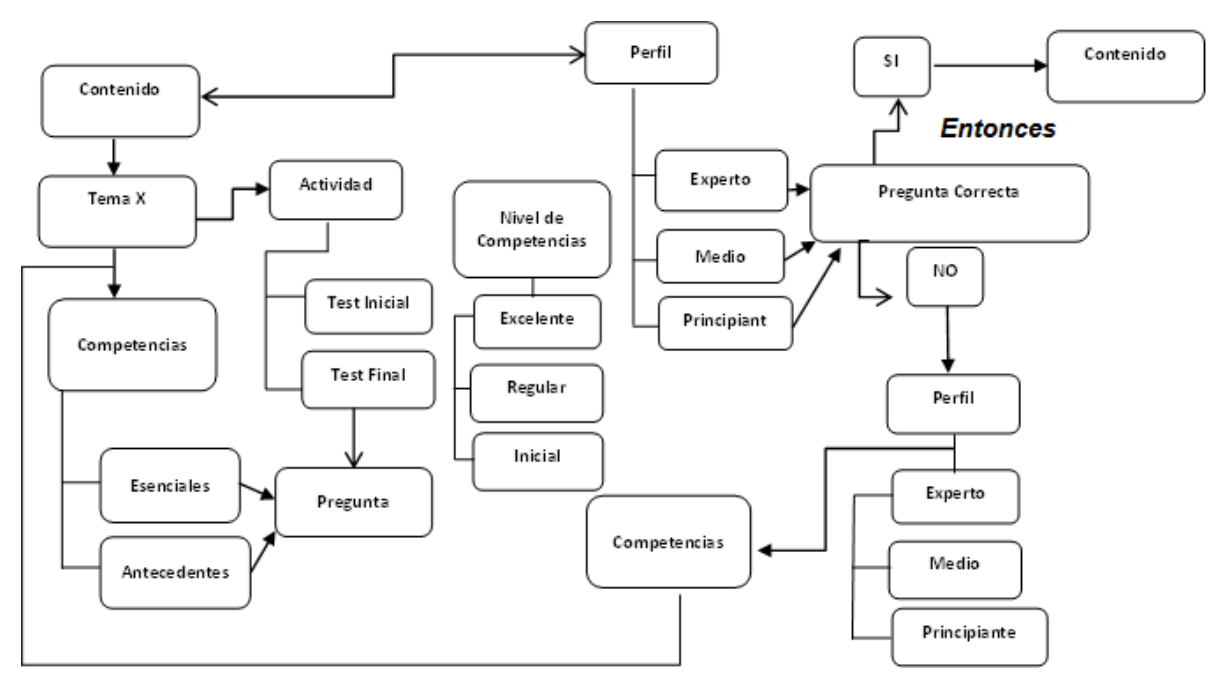

Fig. 3. Diagrama de Reglas para el Diagnóstico del SHA

Para la información del seguimiento académico se utilizó una base de datos en SQL Server, como se muestra en la Figura 4.

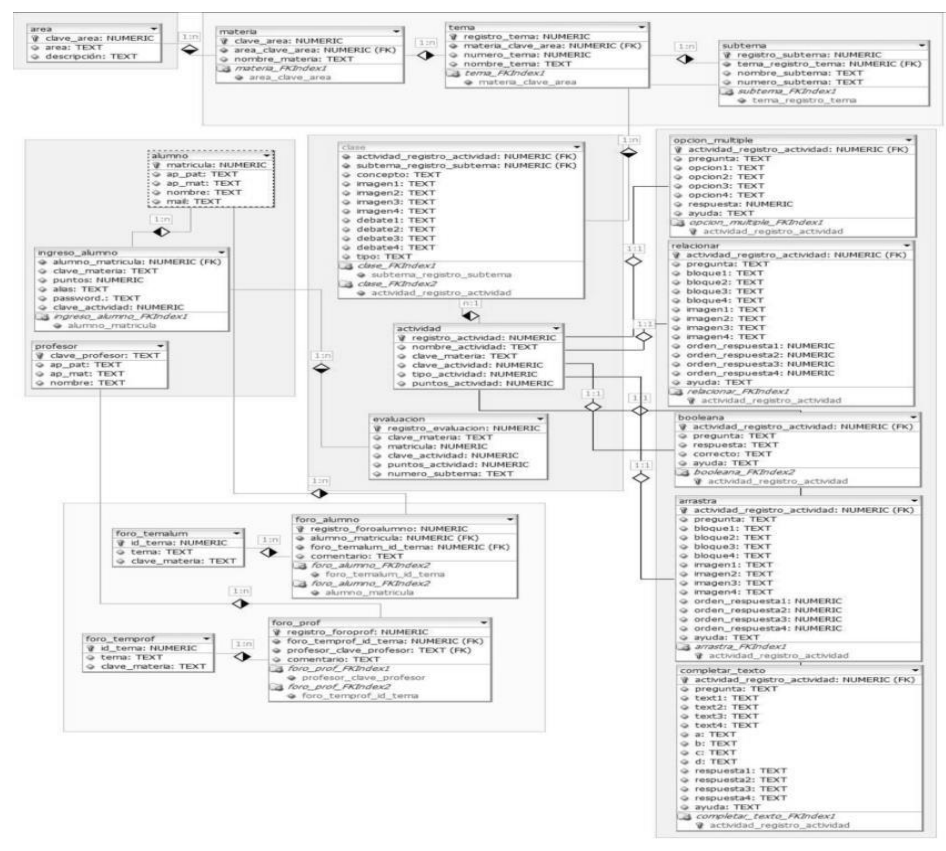

Fig. 4. Modelo de la Base de Datos del Sistema 
De acuerdo a lo anterior el sistema se ha adaptado el contenido del tema de acuerdo al perfil de evaluación diagnosticado con lo cual se definieron:

-Modelo de Usuario: Representar la relación de los usuarios (alumnos) con los contenidos del tema, para lo cual fueron almacenados en una Base de Datos con aspectos relevantes de éste, como: datos personales, clases, actividades, puntuación y el diagnóstico del avance entre otra información. Como se puede ver en la Figura 4.

-Modelo de Dominio: Para obtener los contenidos de aprendizaje se utilizó una de las estructuras hipermediales que permite ir paso a paso los niveles necesarios de aprendizaje para que el alumno revise las clases, actividades, recursos y revisar las deficiencias de su aprendizaje.

-Modelo de Adaptación: Esta parte contiene la descripción de la forma en que se adapta el contenido, los recursos y las actualizaciones de su aprendizaje, para lo cual se basó en reglas Activas, que proponen una separación entre la parte del comportamiento y las entidades del modelo En esta investigación se utilizan reglas de la forma: "Con la ocurrencia de un evento E, si la condición C se cumple, entonces el sistema ejecuta una acción A."

Lo más importante es obtener estas reglas que aporten un conjunto de reglas para el manejo los contenidos y la evaluación de las competencias.

\section{Desarrollo y pruebas del sistema hipermedia basado en competencias para el diagnóstico del aprendizaje de fracciones}

Para desarrollo del sistema educativo SEMATFRAC se usó la herramienta denominada Active Server Pages (ASP) siendo un entorno de secuencias de comandos del servidor que se puede utilizar para crear páginas Web dinámicas o para generar robustas aplicaciones web. Las páginas ASP son archivos de texto con la extensión asp que contienen etiquetas HTML, texto y comandos de secuencias. Pueden llamar a componentes ActiveX para que realicen tareas, como la conexión con bases de datos o cálculos comerciales.

El SHA se implementó en Windows Server 2003 con IIS ver6 y el uso de páginas activas que permite el uso de diferentes scripts y componentes en conjunto con HTML, para mostrar páginas generadas dinámicamente, la tecnología Active Server Pages son un ambiente de aplicación abierto y gratuito en el que se puede combinar código HTML, scripts y componentes ActiveX del servidor para crear soluciones dinámicas y poderosas para la Web [13]. El sistema SMCDAFRAC, inicia entrando a la escuela virtual. Al elegir la opción AULA, el alumno podrá seleccionar la materia de Matemáticas con el tema de FRACCIONES y comenzar su recorrido por el sistema. 
El sistema educativo presenta un menú de opciones en el que se determina el rol que le corresponde al usuario en el sistema (Ver figura 5) y de acuerdo al subtema se presentan las actividades y recursos para la clase.

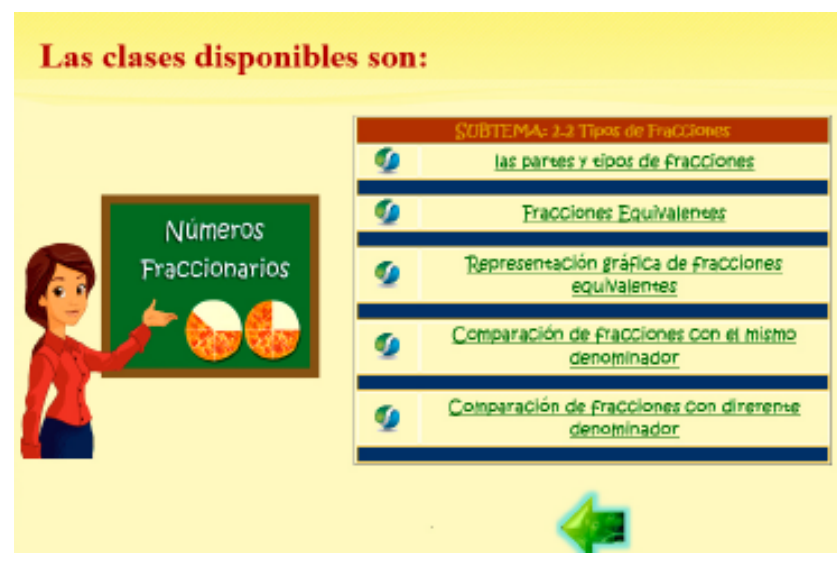

Fig. 5. Menú del Sistema

El nivel de competencias se revisa mediante el diagnostico iniciando con una actividad lo cual permite determinar los aprendizajes y verificar el perfil del alumno (ver Figura 6 y 7 ).

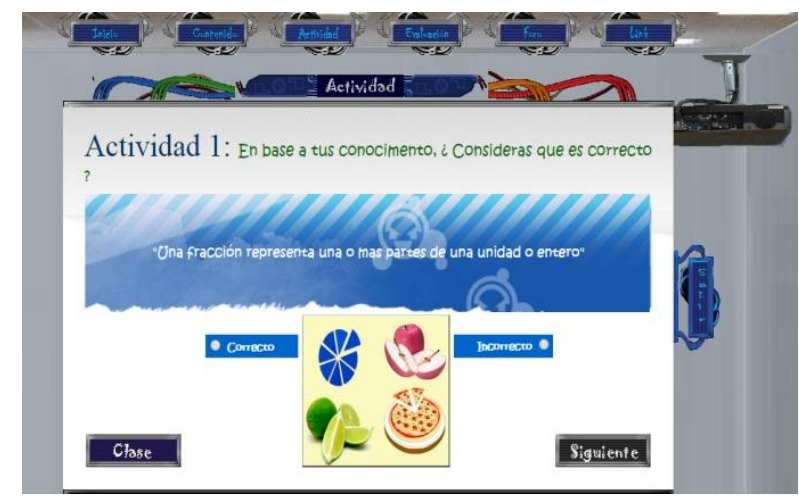

Fig. 6. Actividad de Diagnóstico

El alumno utiliza diferentes recursos donde se le explican conceptos y/o procedimientos que necesita aprender de acuerdo a las estrategias de aprendizaje con las que fueron diseñadas cada actividad [14]. El sistema tiene videos y animaciones para motivar a realizar las actividades y las evaluaciones. 


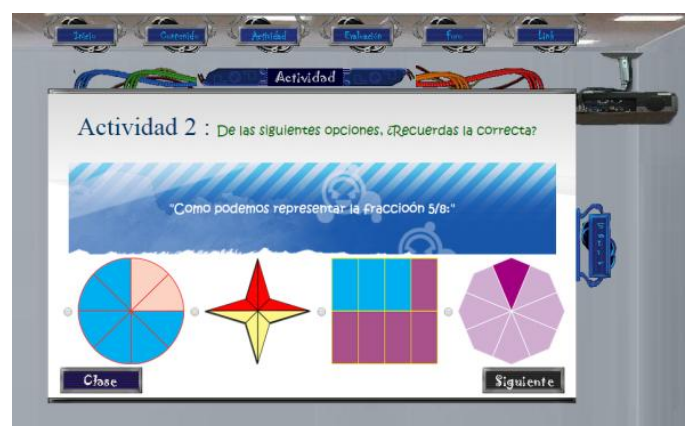

Fig. 7. Actividad de Aprendizaje Esencial

\subsection{Prueba Piloto del Sistema Hipermedia basado en Competencias para el dia- gnóstico del aprendizaje de Fracciones.}

El sistema fue piloteado con alumnos de tercero de primaria a una muestra $n=40$ cuyo objetivo fue obtener el diagnóstico inicial y final. Las evaluaciones permitieron revisar el nivel de logro del desarrollo de las competencias en matemáticas que adquirieron por los alumnos durante el ciclo escolar 2012-2013, siendo el sistema una herramienta de apoyo utilizada con los alumnos de forma presencial durante las sesiones de clases. Las cuatro competencias que se midieron son: Resolver problemas de manera autónoma, Comunicar información matemática, Validar procedimientos y resultados y Manejo de técnicas eficientes donde cada una representa un $25 \%$ del total de las competencias y a partir de esta información es como se realiza la evaluación.

La competencia que más se desarrolló fue "Resolver problemas de manera autónoma" con un $75 \%$ de desarrollo, de $10 \%$ a un $85 \%$ y la segunda fue "El manejo de técnicas eficientes" con un $65 \%$, logrando alcanzar de un $25 \%$ a un $90 \%$ esto conlleva que el uso de un SHA propicia un aprendizaje más significativo en el tema de fracciones, como se muestra en la Tabla1.

Tabla 1. Resultados del Test Inicial, Test Final y Aportación del desarrollo para el Desarrollo de Competencias mediante el SHA

\begin{tabular}{lccc}
\hline \multicolumn{1}{c}{ Competencias Matemáticas } & $\begin{array}{c}\text { Test } \\
\text { Inicial }\end{array}$ & $\begin{array}{l}\text { Test } \\
\text { Final }\end{array}$ & $\begin{array}{l}\text { Uso } \\
\text { Sistema }\end{array}$ \\
\hline Resolver problemas de manera autónoma & $10 \% /$ & $85 \%$ & $75 \%$ \\
Comunicar información matemática & $45 \%$ & $90 \%$ & $45 \%$ \\
Validar procedimientos y resultados & $20 \%$ & $80 \%$ & $60 \%$ \\
Manejo de técnicas eficientes & $25 \%$ & $90 \%$ & $65 \%$ \\
\hline
\end{tabular}

A partir de lo anterior se identificó que el promedio de evaluación fue de $86.25 \%$, y se clasificaron los niveles de aprendizaje de acuerdo a los puntajes del Test final. 
El nivel de desarrollo de competencias que los alumnos adquirieron con respecto al tema de fracciones, donde la mayoría se encuentra en el nivel "Regular", que demuestra que adquirieron las habilidades esenciales e indispensables para la vida.

Por otra parte el nivel de excelente identifica a los alumnos con mayor dominio de las competencias siendo solo el $10 \%$ de la muestra. Tal como se presenta en la Tabla 2.

Tabla 2. Resultados obtenidos del Nivel de Competencias de los Alumnos

\begin{tabular}{lrrr}
\hline \multicolumn{1}{c}{ Competencias Matemáticas } & \multicolumn{3}{c}{ Nivel de Competencia } \\
& Inicial & Regular & Excelente \\
\hline Resolver problemas de manera autónoma & $15 \%$ & $60 \%$ & $10 \%$ \\
Comunicar información matemática & $10 \%$ & $70 \%$ & $10 \%$ \\
Validar procedimientos y resultados & $30 \%$ & $40 \%$ & $10 \%$ \\
Manejo de técnicas eficientes & $10 \%$ & $70 \%$ & $10 \%$ \\
\hline
\end{tabular}

\section{Conclusiones}

Una de las principales contribuciones del Sistema Hipermedia basado en Competencias para el diagnóstico del aprendizaje de Fracciones Matemáticas (SMCDAFRAC) es la adaptación de distintos perfiles de usuarios al aplicar el diagnóstico y evaluación de aprendizajes para establecer recorrido de contenidos y recursos de acuerdo a las necesidades de aprendizaje del alumno.

El SMCDAFRAC promueve en los estudiantes un aprendizaje contextual, experimental, participativo y de autoaprendizaje. El historial del alumno permite identificar sus deficiencias en el tema para establecer estrategias para mejorar su desempeño académico y desarrollar las competencias matemáticas.

El Sistema Hipermedia permite que el estudiante desarrolle las competencias disciplinares en las matemáticas de acuerdo a los nuevos programas educativos del 2011 y a apoyar a los docentes, ya que motiva las competencias tecno-pedagógicas al incluir el uso de las TIC en su práctica docente.

Con base a la información obtenida durante la interacción del alumno con el sistema, es importante observar que se fomentó el trabajo en equipo, la participación y el aprendizaje autónomo.

Una de las principales perspectivas de este trabajo es elaborar Sistemas Hipermedias adaptativos en otros niveles educativos: educación media superior y superior, integrando nuevas tecnologías como son los agentes inteligentes que logren tareas más 
específicas y puedan establecer relaciones con otros aspectos del aprendizaje (estilos de aprendizaje, estrategias de estudio, etc.).

Así también se propone diseñar y desarrollar sistemas hipermedias adaptativos en diferentes asignaturas de acuerdo a los planes y programas de estudios vigentes, para apoyar el desarrollo de las competencias que son evaluadas por distintas pruebas internacionales, para contribuir a mejorar los resultados y la calidad de la educación en México.

\section{Referencias}

1. Coll, C.: Psicología de la educación y prácticas educativas mediadas por las tecnologías de la información y la comunicación: una mirada constructivista. Sinéctica, http://virtualeduca.org/ifdve/pdf/cesar-coll-separata.pdf

2. Navales, O.: Las tecnologías de la información y la comunicación y su impacto en la educación, Universidad Autónoma del Estado de Hidalgo, México (2005).

3. Subsecretaría de Educación Básica. Reforma de la Educación Básica en México. SEP, México (2011).

4. Brusilovsky, P.: Methods and techniques of adaptive hypermedia. User Modeling and User Adapted Interaction, v 6, 2-3, 87-129, Netherlands (1996).

5. Brusilovsky, P., Millan, P.: User Models for Adaptive Hypermedia y Adaptive Educational Systems. The Adaptive Web, Lecture Notes in Computer Science 4321, Berlin: SpringerVerlag, pp. 3-53 (2007).

6. Gaudioso, E.: Contribuciones al Modelado del Usuario en Entornos Adaptativos de Aprendizaje y Colaboración a través de Internet mediante técnicas de Aprendizaje Automático. Tesis Doctoral. Madrid (2002).

7. Raad, H., Causse, B.: "Modeling of an Adaptative Hypermedia System Based on Active Ru-les Springer-Verlag, 2002, pp. 149-157 (2002).

8. Bartolomé, A.: Multimedia interactivo y sus posibilidades en educación superior. Pixel-Bit 1994, no. 1, http://www.sav.us.es/pixelbit/pixelbit/articulos/n1/n1art/art11.htm

9. Gutierrez, L. Martinez, E., Nebreda, T.: Las competencias básicas en el área de Matemáticas, 2008

10. Larman, C.: UML y patrones Introducción al Análisis y Diseño Orientado a Objetos. Pearson Education, España. (2002)

11. F. Grimón, "Tesis doctoral: Modelo para la gestión de dominios de contenido en sistemas hipermedia adaptativos aplicados a entornos de educación superior semipresencial", Universidad de Barcelona, España, 2008, pp. 25-35.

12. Quesada, C, Sánchez, J.: Calificación y Diagnóstico del Aprendizaje por Computadora. México: Noriega Editores (LIMUSA).1996

13. Web Design and Application, http://www.w3.org/standards/webdesign W3C (2013).

14. Peñalosa, E.: Estrategias docentes con tecnologías, Pearson Educación de México, México (2013). 